\title{
Superwetting and aptamer functionalized shrink-induced high surface area electrochemical sensors.
}

\author{
A. Haukea, L.S. Selva Kumara, M. Y. Kimb, J. Peganb, M. Khine ${ }^{b}$, H. Lic, K. W. Plaxcoc, J. Heikenfelda* \\ ${ }^{a}$ Novel Devices Laboratory, School of Electronics and Computing Systems, University of Cincinnati, Cincinnati, Ohio 45221, USA \\ ${ }^{\mathrm{b}}$ Department of Biomedical Engineering, University of California, Irvine, Irvine, California 92697, USA. \\ ${ }^{\mathrm{C}}$ Department of Chemistry and Center for Bioengineering, University of California, Santa Barbara, Santa Barbara, California 93106, USA \\ *email: heikenjc@ucmail.uc.edu
}

\begin{abstract}
Electrochemical sensing is moving to the forefront of point-of-care and wearable molecular sensing technologies due to the ability to miniaturize the required equipment, a critical advantage over optical methods in this field. Electrochemical sensors that employ roughness to increase their microscopic surface area offer a strategy to combatting the loss in signal associated with the loss of macroscopic surface area upon miniaturization. A simple, low-cost method of creating such roughness has emerged with the development of shrink-induced high surface area electrodes. Building on this approach, we demonstrate here a greater than 12-fold enhancement in electrochemically active surface area over conventional electrodes of equivalent on-chip footprint areas. This twofold improvement on previous performance is obtained via the creation of a superwetting surface condition facilitated by a dissolvable polymer coating. As a test bed to illustrate the utility of this approach, we further show that electrochemical aptamer-based sensors exhibit exceptional signal strength (signal-to-noise) and excellent signal gain (relative change in signal upon target binding) when deployed on these shrink electrodes. Indeed, the observed $330 \%$ gain we observe for a kanamycin sensor is 2-fold greater than that seen on planar gold electrodes.
\end{abstract}

Keywords: superwetting, aptamer, sensors, miniature, point-of-care, shrink electrode

\section{Introduction}

Growth in the popularity of point-of-care and wearable devices has ignited significant research into miniaturized electrochemical sensor technologies (Bandodkar and Wang, 2014; Kimmel et al., 2012; Heikenfeld, 2016). The attraction to this class of sensors for such applications is due to their capabilities of being reagentless, single step, wash free, and reversible. Electrochemical sensors are not, however, without potentially important limitations. As with any electronic device, designers are confronted with signal-to-noise constraints, a problem that only multiplies with miniaturization (less signal), increased background noise due to less-sophisticated portable electronics, and increased electromagnetic interference faced for portable and wearable devices. Researchers in this field will thus need to develop novel innovations to solve the signal-to-noise ratio problem for miniaturized sensors.

An electrochemical sensor's performance is highly dependent on the surface area of its working electrode that interfaces with the sample, rendering their miniaturization problematic. Research in the use of "high surface area" electrodes presents a clever solution to this problem in which one creates a rough micro- and/or nanostructured surface topography that considerably enhances the working electrode's electrochemically active surface area (EASA) relative to its macroscopic footprint. While complex chemical deposition methods to this end have been available for decades (Salvarezza et al., 1990; Gabardo et al., 2015; Sonney et al., 2015), recent research has shown that enhanced EASA can be achieved more simply by heat-shrinking a polymer substrate coated with metal thin film electrodes (Gabardo et al., 2013; Pegan et al., 2013). Fabrication of these shrink electrodes is low-cost and does not require sophisticated equipment or clean-room facilities.

The benchmark EASA improvement for shrink electrodes was set by Pegan et al. who observed a 6.6-fold increase in electrochemical signal per macroscopic unit area for such electrodes (Pegan et al., 2013). Alone this significant result is evidence of the potential shrink electrodes offer lab-on-chip (LOC) and point-of-care (POC) applications, but has their full potential been reached? For these polyolefin substrate devices, shrinking reduces the footprint area of the gold film electrodes by about a factor of 20 . The EASA enhancements associated with shrinking should thus theoretically also reach 20 -fold. While constraints such as "secondary wrinkling" (Gabardo et al., 2013) exist that prevent these first-generation devices from reaching the theoretical EASA limit, the substantial gap between demonstrated results and theoretical predictions poses a looming question as to whether the full advantage of the surface topography is being exploited.

Poor wettability leads to non-ideal EASA gains when air bubbles trapped in the wrinkled structure prevent the sample solution from accessing the maximum available surface area (Freschauf et al., 2012) (Fig. 1a). In response we present here a superwetting (Drelich and Chibowski, 2010) surface modification via the addition of a dissolvable polymer coating (Fig. 1b) which extends shrink electrode EASA enhancement by 2 -fold. Using such modified shrink electrodes to fabricate electrochemical aptamer-based (E-AB) sensors we find not only greatly improved signal-to-noise ratios, but also a large and unexpected increase in their signal gain (the change in relative signal upon binding saturating target). Specifically, we employed a kanamycin aptamer into the sensor platform that, in addition to the EASA enhancement, also exhibited a 2.2-fold signal gain improvement over the same E-AB sensors deployed on traditional electrodes. This work provides insights into wetting of structured surfaces and provides a new best-in-class approach for shrink electrode sensors.

\section{Experiment}




\subsection{Design}

Devices were designed to have seven hexagonal electrodes with $360 \mu \mathrm{m}$ side lengths, and were packed in a two row array spaced $50 \mu \mathrm{m}$ apart. $300 \mu \mathrm{m}$ wide trace connections were routed together with $1000 \mu \mathrm{m}$ pitch. Fig. 2 a provides a picture of a completed device with these dimensions. A Mylar photomask of the design was drafted (AutoCAD 2015) and printed by CAD/Art Services, Inc.

\subsection{Bare-gold device fabrication (for EASA measurement only)}

Shrink electrode fabrication closely followed the procedures as those of previous work (Pegan et al., 2013). Polyolefin films (0.5 mil seven layer polyethylene/polypropylene blend, Sealed Air Corp.) were cut into $70 \mathrm{~mm}$ x $70 \mathrm{~mm}$ squares and adhered to silicon wafer substrates using $70 \%$ ethanol and Kapton tape to seal the edges. $40 \mathrm{~nm}$ of gold was then sputter deposited (Q150R S, Quorum Technologies) onto each sample. The samples were subsequently photopatterned using Shipley 1827 (Microchem) positive resist followed by UV exposure (MA6, Suss Microtec) through a photomask. After development, the wafers were wet etched in $\mathrm{I}_{2}: \mathrm{KI}_{\mathrm{H}} \mathrm{H}_{2} \mathrm{O}$ (1:4:40) gold etchant to remove the areas of gold that were exposed through the photolithographic patterning. Finally, acetone was used to remove the remaining photoresist. Once patterned, the PO films were carefully removed from the silicon wafer backing.

Polytetrafluoroethylene (PTFE) was added to electrically insulate the long gold electrodes leading to the hexagonal gold sensor surfaces (Fig. 2a). This same PTFE coating also prevented wicking of fluid along the electrode leads (and therefore ensured that the tested sensor area is constant during testing). To achieve this coating, fluoropel solvent (Cytonix PFC-160) with mass ratio of $0.1 \%$ Teflon AF1600 (Dupont) was applied to electrical trace areas using a cotton swab. After allowing the solvent to dry for 30 min the devices were then shrunk by oven baking at $150{ }^{\circ} \mathrm{C}$ for 5 min (Gabardo et al., 2013; Pegan et al., 2013).

\subsubsection{Polyvinyl pyrolidone superwetting device fabrication and characterization}

Aqueous solution with a mass ratio of $6 \%$ polyvinyl pyrolidone (Sigma-Aldrich PVP10) and $0.01 \%$ non-polar surfactant (Triton $\mathrm{X} 100$ ) was prepared by stirring for $5 \mathrm{~h}$. Approximately $2 \mu \mathrm{L}$ of solution was applied to each exposed electrode area using a pipet such that the surface was nearly covered. The device was immediately placed in vacuum (Thermo Scientific Lindberg Blue M) at $27 \mathrm{in}$. Hg (gauge) for approximately 5 min until noted air bubble formation on the meniscus of PVP solution was observed to cease. Excess solution was then hand removed by wicking into a paper towel. The result was an even coating of clear PVP with all of the wrinkles filled without gas pockets, as seen by comparing Fig. $2 \mathrm{~b}$ and $2 \mathrm{c}$.

A three electrode electrochemical cell with a Pt wire counter (BASi MW-1032) and Ag/AgCl reference electrode (eDAQ ET072) was used to obtain cyclic voltammagrams in $0.1 \mathrm{M} \mathrm{H}_{2} \mathrm{SO}_{4}$ (Fisher Scientific $\mathrm{H}_{2} \mathrm{SO}_{4}$ ). PVP coated shrink electrodes were compared to a commercial gold disk electrode (eDAQ ET076) as working electrodes in the cell. Cyclic voltammetry (CV) scans were run from $0.2 \mathrm{~V}$ to $1.7 \mathrm{~V}$ at a scan rate of $20 \mathrm{mV} / \mathrm{s}$ (PalmSens3). The reduction peaks of the fourth CV scans were integrated using PSTrace 4.6 software. Reduction peak integrals of the CV scans were converted into power densities by dividing by the given electrodes footprint area. Gain in EASA was determined by the ratio of the power densities of PVP coated shrink electrodes to the bulk gold.

\subsubsection{E-AB sensor fabrication and characterization}

Electrodes were immersed in $0.5 \mathrm{M}$ sulfuric acid and pulsed between $0.0 \mathrm{~V}$ and $2.0 \mathrm{~V}$ vs $\mathrm{Ag} / \mathrm{AgCl}$ for 400,000 cycles using the chronoamperometry technique. Each pulse lasted for $2 \mathrm{~ms}$ with no quiet time. This standard preparation procedure was performed to clean the gold surface as well as further roughen it to facilitate coverage by the DNA probes. All probe oligonucleotides, 5'-HO$\left(\mathrm{CH}_{2}\right)_{6}$-S-S- $\left(\mathrm{CH}_{2}\right)_{6}$-GGGACTTGGTTTAGGTAATGAGTCCC- $\left(\mathrm{CH}_{2}\right)_{7}$-methylene blue-3', were dissolved with Tris buffer to a final concentration of $100 \mu \mathrm{M}$, aliquoted and stored at $-20{ }^{\circ} \mathrm{C}$ ready for use. To fabricate our sensors, the freshly cleaned electrodes were immersed in $200 \mathrm{nM}$ DNA solution for $1 \mathrm{~h}$ at room temperature, which was previously prepared by incubating a solution of $100 \mu \mathrm{M}$ thiolated DNA and $20 \mathrm{mM}$ TCEP (tris-(2-carboxyethyl) phosphine hydrochloride (1:200) for $1 \mathrm{~h}$ at room temperature, and further diluted by PBS buffer ( $\mathrm{pH}$ 7.0). The resulting sensors were washed with deionized water and then incubated in $20 \mathrm{mM} 6-$ mercaptohexanol solution overnight at $4{ }^{\circ} \mathrm{C}$. The functionalized sensors were then rinsed with pure water prior to use.

Electrochemical measurements were performed at room temperature using a CHI630C potentiostat with a CHI684 Multiplexer $(\mathrm{CH}$ Instruments, Austin, TX) and a standard three-electrode cell containing a Pt counter electrode and a $\mathrm{Ag} / \mathrm{AgCl}(3 \mathrm{M} \mathrm{NaCl})$ reference electrode. Square wave voltammetry was performed using a potential window of $-0.10 \mathrm{~V}$ to $-0.4 \mathrm{~V}$ (versus $\mathrm{Ag} / \mathrm{AgCl}$ ), potential step of $1 \mathrm{mV}$, and amplitude of $0.05 \mathrm{~V}$ at a frequency of $500 \mathrm{~Hz}$. Curve fitting of data was performed using Microsoft Excel software.

\section{Device operation theory}

Deep areas of the shrunken surface trap air very efficiently when interfacing aqueous liquids (Fig. 1a). As air does not readily dissolve into water, this structure diminishes the total EASA. Others have leveraged this property as an advantage in developing antibacterial surfaces (Freschauf et al., 2012), but it is a major complication for electrochemical sensing applications and the limiting factor for the EASA improvements. Fig. 1b depicts the proposed superwetting enhancement by simply replacing the air and filling 
the wrinkles with PVP. In this scenario, aqueous sample is incident on a hydrophilic surface that it can fully wet against. As PVP dissolves, it diffuses into the bulk solution while the sample in turn flows in to fill the space left by the PVP. This modification allows sample to access more electrode surface and maximize the EASA gain achieved by shrinking.

For validation, E-AB sensors were also utilized (Biagiotti et al., 2012), which can work continuously even in whole blood. E-AB sensors are based on DNA folding that occurs when an analyte is bound. The folding event then changes the distance between a redox couple tag and a gold electrode surface, which changes the amount of charge transfer between the redox couple and the electrode (Pheeny \& Barton, 2012). Mechanisms for charge transfer are localized at the surface of the electrode, allowing E-AB sensors to operate with low susceptibility to surface fouling and without reagents (Heikenfeld, 2016).

\section{Results}

Footprint areas for the gold disk and polyvinyl pyrolidone (PVP) coated shrink electrodes were measured to be $0.79 \mathrm{~mm}^{2}$ and $0.31 \mathrm{~mm}^{2}$ respectively. CV curves for these electrodes are shown in Fig. 3. Using the CV data and measured areas, the integrated reduction peak power densities were determined to be $0.367 \mu \mathrm{AV} / \mathrm{mm}^{2}$ and $4.67 \mu \mathrm{AV} / \mathrm{mm}^{2}$ respectively, indicating a 12.7 -fold enhancement in EASA with the PVP coated shrink electrode. The result increases upon the previous 6.6-fold result observed (Pegan et al., 2013) by a factor of 1.9. This greater than 12 -fold gain was repeatable. To verify the results, two separate control CV scans were run using the gold disk electrode (supplementary data provided in Appendix A Fig. A.1). One was run in $0.1 \mathrm{M} \mathrm{H}_{2} \mathrm{SO}_{4}$ solution and the other in bulk $6 \%$ PVP, $0.01 \%$ Triton X100 solution used to coat the shrink electrodes. The former produced a voltammagram characteristic of $\mathrm{H}_{2} \mathrm{SO}_{4}$ while the pure coating voltammagram showed insignificant redox activity in the $\mathrm{H}_{2} \mathrm{SO}_{4}$ reduction peak range around $0.85 \mathrm{~V}$, indicating that the amplification from the PVP coated shrink electrodes was due to the increased wetting on the surface and not a response to the dissolved coating in the sample.

Square wave voltammagrams for the E-AB sensor are shown in Fig. 4a. As expected the methylene blue reduction peak at -0.28 $\mathrm{V}$ increases significantly with increasing kanamycin concentrations. The same data presented as a target titration curve produces the monotonic Langmuir isotherm behavior expected for this type of binding event, yielding a dissociation constant of $2.0 \mathrm{mM}$ (Fig. $4 \mathrm{~b}$ ). Also of note, the plot shows the $330 \%$ signal change seen upon saturating target represents a $>2$-fold improvement over that seen for the same E-AB sensor when fabricated on a traditional, macroscopic gold electrode (Fig. 4b). Therefore, in addition to a total signal increase due to increase in surface area, the signal change (response) of the E-AB sensor has also increased. The origins of this improvement are unclear but are reproducible. The resulting increase in sensitivity (to small changes in target concentration) for the shrink electrode is easily seen in the difference in slopes of the curves in their pseudo-linear regions. The detection capability is within the 2-6 $\mu \mathrm{M}$ range that is clinically relevant for aminoglycoside concentrations (Rowe, et al., 2010; Setia and Gross, 1976) (supplementary data provided in Appendix A Figure A.2). An equilibration time of 2 min (time to achieve $90 \%$ of maximum signal) was observed at $1 \mathrm{mM}$ target concentrations (supplementary data provided in Appendix A, Fig. A.3). This is slightly slower than the equilibration kinetics seen with planar electrodes, presumably due to slower diffusion associated with the greater topological complexity of the wrinkled surface.

It should be noted that the self-assembled monolayer (SAM) of thiol required for E-AB sensing presented a very effective superwetting modification by itself. In fact, sample solution uncontrollably wet over all areas of the completed devices. Therefore the PTFE coating was added to confine the sample solution to the electrode areas of the devices and no dissolvable polymer was necessary for superwetting of the surface for the E-AB sensors.

\section{Discussion}

The PVP coating superwetting strategy produces a nearly two-fold increase in EASA over shrinking alone. However, the 12.7fold increase in current seen when shifting from a planar electrode to a PVP coated shrink electrode still indicates that there is potential for further enhancement toward the 20 -fold theoretical maximum. Further optimization of this process would likely lead to even better results. For instance, PVP can be replaced by a number of dissolvable polymers such as poly-vinyl alcohol or sucrose. The choice of coating will ultimately be application specific to the end user based on compatibility with intended detection target and other sample constituents. Another optimization strategy could be minimization of polymer film thickness which could aid the sample solution in entering more confined areas of the wrinkled surface more rapidly. Small volume samples could also benefit from this by limiting the possibilities of interference from a relatively large amount of PVP as compared to target solution sample.

Photolithography was used to produce the sputter mask for gold patterning in this instance due to the close packing of the electrode array. It should be noted that this is not necessary in all applications and could be simplified by use of shadow masks that can be patterned using a variety of desired methods. Another fabrication process improvement would be to develop a pre-shrinking PVP coating method. This change would eliminate the vacuum degas step and improve the manufacturing scalability for future commercial development. Thus Triton X100 would also no longer be required as this surfactant was added solely to assist the PVP solution in wetting to the gold surface in the presence of trapped air. This method should be feasible judging from the PTFE coating step which showed no observable effects on the final shrunk micro-scale surface topography upon visual inspection. However, more advanced inspection techniques such as microscopy imaging or electrochemical methods would be required to verify that the nanoscale secondary structuring was not adversely affected.

The $330 \%$ signal gain seen for E-AB kanamycin sensors built on shrink electrodes is significantly greater than that seen on planar electrodes, which is more typically only $150 \%$ (Fig. 4b). Higher gain generally translates into improved measurement precision as it 
enables robust detection at lower fractional receptor occupancy. The origins of this higher gain are unclear, as the enhanced surface area (and thus signaling current) produced by nanoscale texturing of the surface should enhance the current produced by both the bound and unbound states of the aptamer receptor, leading to no net change in relative gain. Further research is thus needed to fully understand the reason for the observed higher gain. In contrast to the improved gain, the binding kinetics of this E-AB sensor were slightly slower, requiring 2 min to reach $\sim 90 \%$ maximum signal (supplementary data provided in Appendix A Fig. A.3), while simple, planar electrodes typically require less than $1 \mathrm{~min}$ to achieve this mark. This is a likely product of restricted advective and diffusive transport in wrinkled structures. However, this drawback is a comparably minor tradeoff with respect to the signal gain enhancement. Whether optimizing the shrink E-AB sensor fabrication parameters could further extend the signal gain as well as boost other performance metrics such as sensitivity and limits of detection remain open questions; the results reported here are offered as a proofof-concept for the validity and possible advantages of using modified shrink electrodes in sensors of this class (Lubin and Plaxco, 2010). Further work will investigate the full causality of the enhancements, optimize performance and explore the extension of the improvements to other aptamer probes.

\section{Conclusions}

Significant improvements were achieved upon the already impressive previously published shrink electrode performance. A 12.7-fold EASA enhancement was achieved with the addition of a dissolvable polymer coating. This enhancement doubles the previous benchmark and can be done with one additional simple fabrication step (PVP coating). Additionally the shrink electrodes far surpassed expectations as an E-AB sensor platform. Its $330 \%$ signal gain is a 2.2-fold improvement upon previously reported results for similar aminoglycoside E-AB sensors recorded using more traditional "flat" electrodes. These advancements further support the candidacy of shrink electrodes for robust, easily fabricated, and low-cost electrochemical sensor solutions in lab-on-chip and point-ofcare applications.

\section{Acknowledgements}

This research was supported in part by an appointment to the Student Research Participation Program at the U.S. Air Force Research Laboratory, $711^{\text {th }}$ Human Performance Wing, Human Effectiveness Directorate, Human-Centered Intelligence, Surveillance and Reconnaissance Division administered by the Oak Ridge Institute for Science and Education through an interagency agreement between the U.S. Department of Energy and USAFRL.

The authors would like to acknowledge support from the National Science Foundation and the industrial members of the Center for Advanced Design and Manufacturing of Integrated Microfluidics (NSF I/UCRC award number IIP-1362165, UCI, and IIP1362048, UC).

\section{Conflict of interest statement}

Corresponding author Jason Heikenfeld and co-author Kevin Plaxco have equity interests in Eccrine Systems, Inc., a company that may potentially benefit from the research results, and also serve on the company's Board and Scientific Advisory Board, respectively. The terms of these arrangements have been reviewed and approved by the University of Cincinnati and University of California-Santa Barbara in accordance with their conflict of interest policies.

\section{Appendix A. Supplemental Material}

Electronic supplementary material is available online.

\section{References}

Bandodkar, A. J., Wang, J., 2014. Non-invasive wearable electrochemical sensors: as review. Trends Biotechnol. 32, 363-371.

Biagiotti, V., A. Porchetta, S. Desiderati, K. W. Plaxco, G. Palleschi, F. Ricci, 2012. Probe accessibility effects on the performance of electrochemical biosensors employing DNA monolayers. Anal. Bioanal. Chem. 402, 413-421.

Drelich, J., Chibowski, E., 2010. Superhydrophilic and Superwetting Surfaces: Definition and Mechanisms of Control. Langmuir 26, 18621-18623.

Freschauf, L. R., McLane, J., Sharma, H., Khine, M., 2012. Shrink-Induced Superhydrophobic and Antibacterial Surfaces in Consumer Plastics. PLoS ONE, 7, e40987.

Gabardo, C. M., Adams-McGavin, R. C., Vanderfleet, O. M., Soleymani, L., 2015. Rapid prototyping of microfluidic devices with integrated wrinkled gold micro-nano textured electrodes for electrochemical analysis. Analyst 140, 5781-5788.

Gabardo, C., Zhu, Y., Soleymani, L., Moran-Mirabal, J. M., 2013. Bench-Top Fabrication of Hierarchically Structured High SurfaceArea Electrodes. Adv. Funct. Mater. 23, 3030-3039.

Heikenfeld, J., 2016. Non-invasive Analyte Access and Sensing through Eccrine Sweat: Challenges and Outlook circa 2016. Electroanalysis 28, 1242-1249. 
Kimmel, D. W., LeBlanc, G., Meschievitz, M. E., Cliffel, D. E., 2012. Electrochemical Sensors and Biosensors. Anal. Chem. 84, 685707.

Lubin, A. A., Plaxco, K. P., 2010. Folding-based electrochemical biosensors: the case for responsive nucleic acid architectures. Acc. Chem. Res. 43, 496-505.

Pegan, J. D., Ho, A. Y., Bachman, M., Khine, M., 2013. Flexibile shrink-induced high surface area electrodes for electrochemiluminescent sensing. Lab Chip 13, 4205-4209.

Pheeny, C. G., Barton, J. K., 2012. DNA Electrochemistry with Tethered Methylene Blue. Langmuir 28, 7063-7070.

Rowe, A. A., Miller, E. A., Plaxco, K. W., 2010. Reagentless Measurement of Aminoglycoside Antibiotics in Blood Serum via an Electrochemical, Ribonucleic Acid Aptamer-Based Biosensor. Anal. Chem. 82, 7090-7095.

Setia, U., Gross, PA, 1976. Administration of tobramycin and gentamicin by the intravenous route every $6 \mathrm{hr}$ in patients with normal renal function. J Infect Dis. 134, S125-129.

Salvarezza, R. C., C. Alonso, J. M. Vara, E. Albano, H. O. Martin, A. J. Arvia, 1990. Monte Carlo simulation applicable to the growth of rough metal overlayers: Parametric relationships related to the electrochemical roughening. Phy. Rev. B: Condens. Matter Mater. Phys. 41, 502-512.

Sonney, S., Shek, N., Moran-Mirabal, J. M., 2015. Rapid bench-top fabrication of poly(dimethylsiloxane), polystyrene microfluidic devices incorporating high-surface-area sensing electrodes. Biomicrofluidics 9, 026501.

(a) no PVP coating

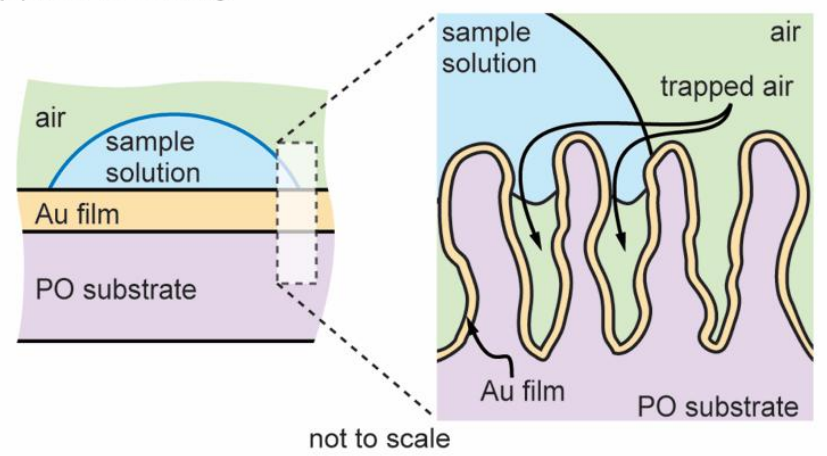

(b) with PVP coating

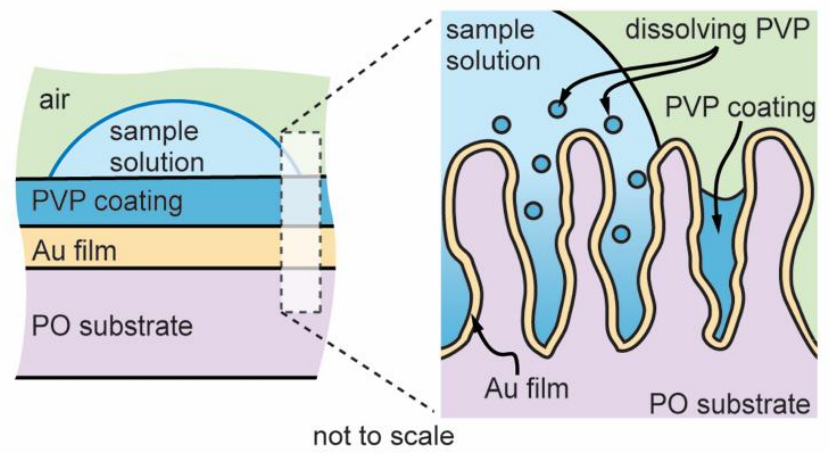

Fig. 1 Cross sectional schematic depictions of EASA optimization strategy (a) bare gold (Au) surface on heat-shrunk polyolefin (PO); (b) a gold surface with a poly-vinyl pyrolidone (PVP) coating. Schematics are not to scale. 
(a) overall device with PTFE coating

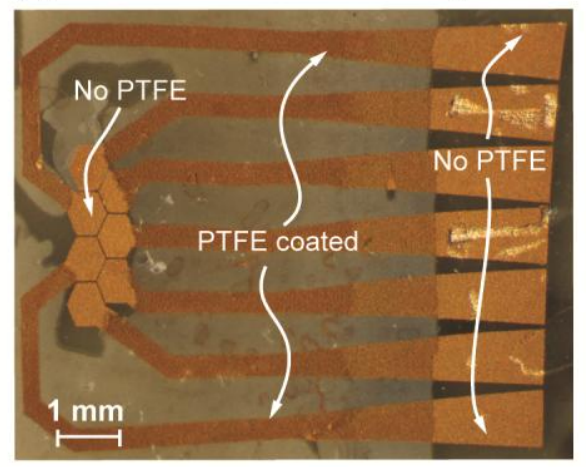

(b) electrode with no PVP coating

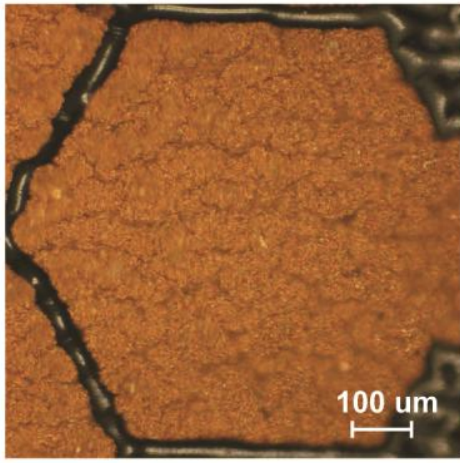

(c) electrode with PVP coating

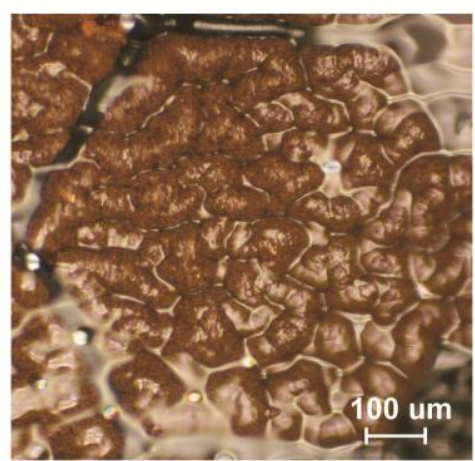

Fig. 2 Photographs of shrink electrodes (a) shrink electrode array after polytetrafluoroethylene (PTFE) coating and shrinking; (b) close up of shrunken bare gold electrode surface, and (c) close up of same shrunken gold electrode in Fig. 2b with added polyvinyl pyrolidone (PVP) coating.

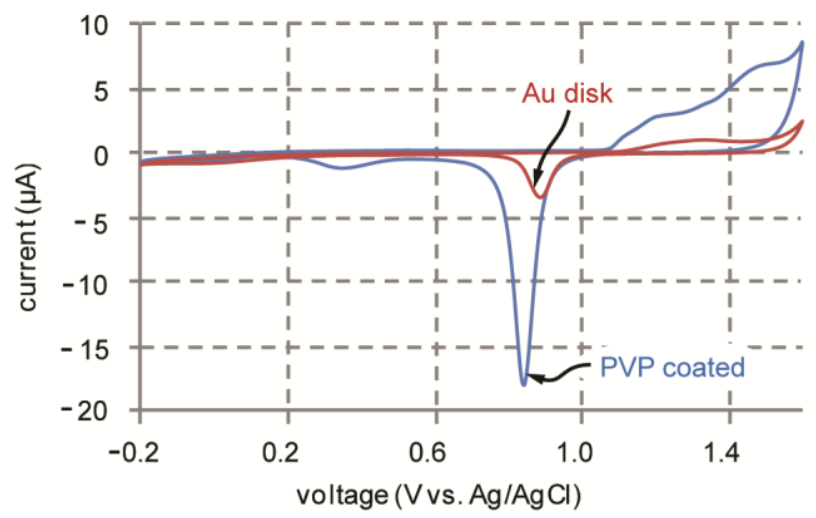

Fig. 3 Cyclic voltammagram comparison of bulk gold $\left(0.79 \mathrm{~mm}^{2}\right.$ footprint $)$ and polyvinyl pyrolidone (PVP) coated shrink electrodes $\left(0.31 \mathrm{~mm}^{2}\right.$ footprint). 
(a) target binding response

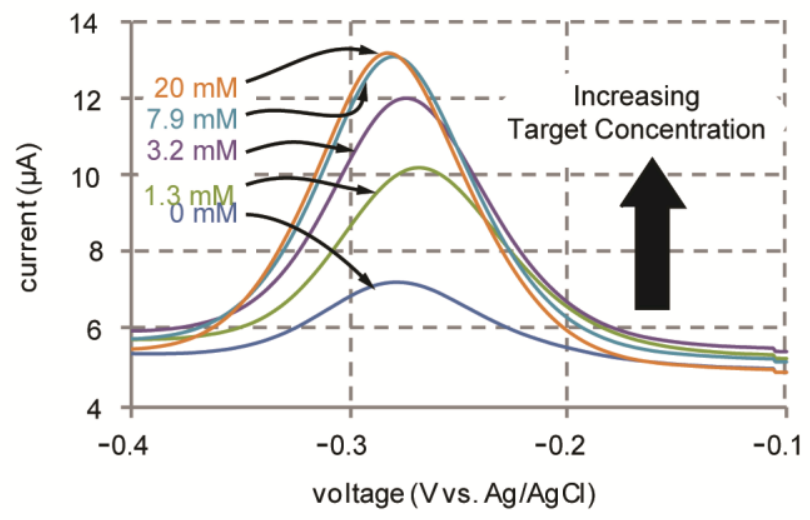

(b) target titration curve

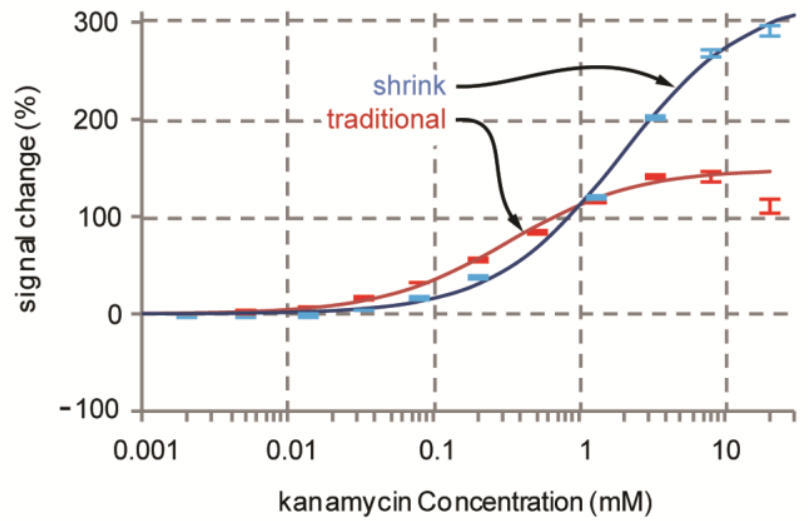

Fig. 4 Electrochemical aptamer based (E-AB) sensor for kanamycin fabricated on a shrink electrode: (a) square wave voltammarams of the sensor in response to increasing amounts of kanamycin; (b) a plot of signal gain versus target concentration fits the Langmuir isotherm and is compared to the curve of a similar E-AB sensor fabricated on a traditional electrode. 